\title{
Enfermagem no Cuidados ao Paciente Neurocirúrgico em uso de Derivação Ventricular Externa: relato de experiência
}

\author{
Nursing in the Care of Neurosurgical Patients using External Ventricular Drain: experience report \\ Enfermería en el Cuidado del Paciente Neuroquirúrgico mediante Bypass Ventricular Externo:
} informe de experiencia

Recebido: 26/07/2021 | Revisado: 07/08/2021 | Aceito: 09/08/2021 | Publicado: 13/08/2021

\author{
Carina Galvan \\ ORCID: https://orcid.org/0000-0003-2111-5432 \\ Hospital de Clínicas de Porto Alegre, Brasil \\ E-mail: cgalvan@hcpa.edu.br \\ Lisiane Paula Sordi Matzenbacher \\ ORCID: https://orcid.org/0000-0002-1407-7717 \\ Hospital de Clínicas de Porto Alegre, Brasil \\ E-mail: 1sordi@hcpa.edu.br \\ Débora Machado Nascimento do Espírito Santo \\ ORCID: https://orcid.org/0000-0003-0533-0335 \\ Hospital de Clínicas de Porto Alegre, Brasil \\ E-mail: dsanto@hcpa.edu.br \\ Rosaura Soares Paczek \\ ORCID: https://orcid.org/0000-0002-4397-1814 \\ Hospital de Clínicas de Porto Alegre, Brasil \\ E-mail: rspaczek@gmail.com \\ Ana Karina Silva da Rocha Tanaka \\ ORCID: https://orcid.org/0000-0003-2488-3656 \\ Hospital de Clínicas de Porto Alegre, Brasil \\ E-mail: anakarinatanaka@gmail.com
}

\begin{abstract}
Resumo
Objetivo: descrever os cuidados de Enfermagem prestados aos pacientes acometidos por acidente vascular cerebral hemorrágico em uso de derivação ventricular externa. Método: estudo descritivo, do tipo relato de experiência, realizado em junho de 2021. O local do estudo é em um hospital público universitário do Sul do Brasil. Resultados: na admissão, cabe ao enfermeiro certificar-se da localização e permeabilidade da drenagem ventricular, posicionando o paciente em decúbito dorsal, com alinhamento esternal e cabeceira a $30^{\circ}$. Para realizar a instalação do sistema, é necessário acoplar o transdutor ao monitor multiparâmetros, sendo que o ponto zero do sistema deve estar alinhado ao meato acústico externo, fazendo uso de régua niveladora. O sistema é então fixado em suporte exclusivo para esta finalidade, mantendo a altura do ponto para o escoamento de líquido cefalorraquidiano em 20 centímetros acima do transdutor ou do ponto zero, considerando a pressão intracraniana normal em até $15 \mathrm{mmHg}$. Quando necessária a mudança na altura da cabeceira do leito, o circuito proximal da cabeça do paciente deve ser interrompido, fechando o clamp, mantendo a bolsa de drenagem bem fixada, evitando alterar nível e drenagem inadvertida. Considerações finais: constata-se a complexidade no cuidado diante do paciente neurocrítico, evidenciando, dessa forma, a necessidade da implantação de intervenções baseadas em planos de cuidados específicos para a obtenção do tratamento adequado.
\end{abstract}

Palavras-chave: Educação em saúde; Cuidados de enfermagem; Acidente vascular cerebral.

\begin{abstract}
Objective: to describe the nursing care provided to hemorrhagic stroke patients using an external ventricular drain. Method: a descriptive study, of experience report type, carried out in June 2021. The study site is in a public university hospital in Southern Brazil. Results: on admission, the nurse is responsible for making sure of the location and permeability of the ventricular drainage, positioning the patient in dorsal decubitus, with sternal alignment and head of the bed at $30^{\circ}$. To install the system, it is necessary to couple the transducer to the multi-parameter monitor, and the zero point of the system must be aligned with the external acoustic meatus, using a leveling ruler. The system is then fixed on a support exclusively for this purpose, maintaining the point height for the cerebrospinal fluid drainage at 20 centimeters above the transducer or the zero point, considering a normal intracranial pressure of up to $15 \mathrm{mmHg}$. When necessary to change the bed headboard height, the proximal circuit of the patient's head should be interrupted, closing the clamp, keeping the drainage bag well fixed, avoiding level changes and inadvertent drainage.
\end{abstract}


Final considerations: the complexity of care for neuro-critical patients is evident, thus highlighting the need for the implementation of interventions based on specific care plans to obtain the appropriate treatment.

Keywords: Health education; Nursing care; Stroke.

\begin{abstract}
Resumen
Objetivo: describir los cuidados de enfermería que se brindan a los pacientes con ictus hemorrágico mediante bypass ventricular externo. Método: estudio descriptivo, tipo relato de experiencia, realizado en junio de 2021. El sitio de estudio se encuentra en un hospital universitario público del sur de Brasil. Resultados: al ingreso le corresponde al enfermero cerciorarse de la ubicación y permeabilidad del drenaje ventricular, colocando al paciente en decúbito dorsal, con alineación esternal y cabeza a $30^{\circ}$. Para instalar el sistema es necesario acoplar el transductor al monitor multiparamétrico, y el punto cero del sistema debe estar alineado con el conducto auditivo externo, utilizando una regla de nivelación. A continuación, el sistema se fija sobre un soporte exclusivo para este fin, manteniendo la altura del punto de flujo de líquido cefalorraquídeo a 20 centímetros por encima del transductor o del punto cero, considerando una presión intracraneal normal de hasta $15 \mathrm{mmHg}$. Cuando sea necesario cambiar la altura de la cabecera de la cama, se debe interrumpir el circuito proximal de la cabeza del paciente, cerrando la pinza, manteniendo bien fijada la bolsa de drenaje, evitando cambios de nivel y drenaje involuntario. Consideraciones finales: existe la complejidad de la atención al paciente neurocrítico, evidenciando así la necesidad de implementar intervenciones basadas en planes de atención específicos para obtener un tratamiento adecuado.
\end{abstract}

Palabras clave: Educación en salud; Atención de enfermeira; Accidente cerebrovascular.

\title{
1. Introdução
}

As doenças cerebrovasculares são a segunda causa de mortalidade no mundo, segundo a Organização Mundial da Saúde (OMS). No Brasil, onde existe uma desinformação sobre seu tratamento, o Acidente Vascular Cerebral (AVC) é a primeira causa de óbito, ultrapassando as doenças coronarianas e causando grande impacto na saúde da população (Barcelos et al., 2016; Ministério da Saúde, 2020).

O Acidente Vascular Hemorrágico (AVCH), também definido como hemorragia intracerebral, caracteriza-se pela ruptura de uma artéria com posterior derrame sanguíneo para o espaço intraparenquimatoso, tem maior incidência na etnia afro-americana e no gênero masculino, porém isto se inverte com o avanço da idade. Entre outros fatores de risco, encontramos a Hipertensão Arterial Sistêmica (HAS), seguido pelo alcoolismo e o tabagismo (Guimarães \& Pereira.,2018; Figueiredo, et al., 2020).

A hemorragia intracerebral frequentemente é extensa e catastrófica, mais fatal do que o acidente vascular cerebral isquêmico, suas manifestações clínicas estão de acordo com a área cerebral afetada, incluindo alterações no nível de consciência, função cognitiva, defeitos no campo visual, na linguagem e as disfunções motoras como a hemiplegia ou a hemiparesia. Cerca de $90 \%$ dos sobreviventes, não recuperam toda a função cerebral perdida, restringindo assim, o desempenho das atividades de vida diária (Gonçalves et al., 2019; Nascimento \& Carvalho, 2020).

A pressão intracraniana (PIC) pode ser definida como a pressão sob a qual os componentes intracranianos: tecido cerebral, líquido cefalorraquidiano (LCR) e vascular são mantidos dentro da caixa craniana, sendo definida como pressão intracerebral normal quando os valores forem de 5 a $15 \mathrm{mmHg}$. Qualquer alteração no volume de algum destes componentes, assim como adição de uma lesão, podem levar a um aumento da PIC, que quando aumentada, acima de $20 \mathrm{mmHg}$, tem importante repercussão fisiológica interferindo na pressão de perfusão cerebral (PPC), comprimindo estruturas cerebrais e podendo causar hérnia cerebral, esta última tendo desfecho fatal. O monitoramento da PIC por meio do implante de um cateter de polietileno na cavidade ventricular acoplado a um dispositivo de pressão transdutor é fundamental para evitar danos cerebrais secundários sendo o único método aceito e seguro para o diagnóstico e tratamento da hipertensão intracraniana. (Roger et al., 2017; Almeida \& Meneguin, 2019; Magalhães et al., 2020).

A derivação ventricular externa (DVE) é considerada padrão ouro no tratamento dos pacientes com patologias neurológicas onde ocorre a hipertensão intracraniana (HIC). A DVE permite a monitorização contínua e auxilia na redução da PIC, através da drenagem do LCR ou sangue pela bolsa coletora (Sakamoto et al., 2021). 
A principal finalidade do cuidado de enfermagem em pacientes neurocirúrgicos é minimizar e prevenir danos decorrentes da HIC, fazendo necessário uma assistência qualificada. A enfermagem atua identificando imediatamente os déficits no tratamento, realizando um planejamento com implementação de plano de cuidados específicos para esta patologia (Szabo et al., 2014; Souza et al., 2021).

Portanto, este estudo tem como objetivo descrever os cuidados de enfermagem prestados aos pacientes acometidos por acidente vascular cerebral hemorrágico em uso de derivação ventricular externa.

\section{Metodologia}

Trata-se de um estudo descritivo do tipo relato de experiência, realizado em junho de 2021, descrito por enfermeiras assistenciais, com experiência em cuidados a pacientes em pós operatório imediato de colocação de derivação ventricular externa. O local do estudo é em um hospital público universitário do sul do Brasil.

O setor possui 10 leitos de recuperação pós anestésica e 10 leitos de terapia intensiva, onde são admitidos pacientes em pós operatório imediato de cirurgias de médio e grande porte; o serviço conta com 01 enfermeira para cada 05 leitos de terapia intensiva e sala de recuperação por turno, um técnico de enfermagem para cada dois leitos críticos e um técnico de enfermagem para cada 03 leitos de sala de recuperação. Os pacientes em pós operatório imediato de cirurgias neurológicas são admitidos no leito de terapia intensiva e são assistidos por uma enfermeira, um técnico em enfermagem e um médico intensivista.

A narrativa descrita como relato de experiência é um método qualitativo que visa descrever as especificidades em detalhes de vivências e experiências de um indivíduo, de modo a clarificar e demonstrar a prática (Daltro \& Farias, 2019). A experiência narrada é singular e depende das reflexões do relator sobre a situação vivenciada, desafiando o desenvolvimento dos conhecimentos técnico-científicos por meio da pesquisa e das relações humanas descritas a partir do narrado e vivido (Minayo, 2012).

\section{Resultados}

A admissão do paciente neurocirúrgico crítico após hemorragia intraparenquimatosa na UTI, necessita do preparo do leito de acordo com o perfil do paciente que será admitido. O enfermeiro da unidade recebe o plantão da enfermeira do bloco cirúrgico, onde são relatadas as condições em que o paciente será transferido para UTI, como a hemodinâmica, presença de tubos, sondas, drenos, catéteres venosos, arteriais, monitorização de sinais vitais, infusões, padrão ventilatório, frequência cardíaca, débito urinário, perdas sanguíneas no transoperatório e antibioticoterapia. A enfermeira, após receber o paciente, deve orientar a equipe de enfermagem a preparar o box e o leito conforme necessidades específicas.

Ao admitir o paciente na UTI cabe ao enfermeiro certificar-se da localização e permeabilidade da DVE posicionando o paciente em decúbito dorsal, alinhamento esternal e cabeceira a $30^{\circ}$. Para realizar a instalação da DVE é necessário acoplar o transdutor ao monitor e o ponto zero do sistema deve estar alinhado ao meato acústico externo, fazendo uso de régua niveladora. O sistema é então fixado em suporte exclusivo para esta finalidade, mantendo a altura do ponto de escoamento de LCR em $20 \mathrm{~cm}$ acima do transdutor ou do ponto zero, considerando a PIC normal em até $15 \mathrm{mmHg}$.

Quando necessárias mudanças na altura da cabeceira do leito, o circuito proximal da cabeça do paciente deve ser interrompido fechando o clamp, mantendo a bolsa de drenagem bem fixada, evitando alterar nível e drenagem inadvertida. A drenagem do LCR é realizada contra o gradiente de pressão hidrostática, ou seja, na dependência da altura onde é instalada a bolsa aberta. O enfermeiro deve registrar em prontuário a quantidade de LCR drenado na bureta coletora a cada 6 horas, esvaziando a mesma, assim como quando a bolsa coletora atingir $2 / 3$ deverá ser desprezado o LCR coletado utilizando técnica 
asséptica. Outro cuidado realizado pelo enfermeiro é a calibração do sistema e a monitorização das condições fisiológicas da onda da PIC a cada 6 horas ou mudança na altura da cama ou graus da cabeceira.

O primeiro curativo na região pericateter é realizado pela equipe médica da neurocirurgia e permanece fechado por 48 horas. A enfermagem deve observar se há saída de líquor através do curativo, inspecionando as condições, presença de sangramento e abaulamentos, fixação, característica e volume das drenagens, realizando o registro no controle assistencial do paciente. A escala de Coma de Glasgow deve ser aplicada e tem por objetivo determinar o score de consciência através da observação do comportamento, onde são avaliadas as pupilas no que se refere a diâmetro, formato, simetria, fotorreação (reação a luz), resposta verbal e resposta motora. A escala tem três variáveis, que podem ser graduadas de 1 a 5 . Sendo assim, escore 3 representa o máximo de gravidade e escore 15 o mínimo.

Quando necessário transportar o paciente deve-se quantificar e esvaziar o volume presente na bureta, para evitar que o líquido e a umidade obstruam o filtro, assim como todos os clamps deverão ser fechados inclusive o do filtro para evitar falhas no sistema.

O registro da PPC é extremamente importante na avaliação do paciente neurocirúrgico com controle da PIC, é um cuidado realizado pela enfermagem através da fórmula: PPC $=$ PAM - PIC, sendo que o valor de PPC deve ser igual ou superior a $70 \mathrm{mmHg}$, pois valores abaixo indicam um fluxo sanguíneo cerebral inadequado.

Outros cuidados que trazem benefícios são a desobstrução de vias aéreas de curta duração, máximo de 15 segundos, podendo esta, ser precedida de pré-oxigenação com FiO2 100\%, limitando desta forma a HIC durante a aspiração das vias aéreas. Um controle da diurese e o decúbito deve ser alternado de $2 / 2 \mathrm{~h}$ com atenção para manter o alinhamento da cabeça com a região torácica esternal, evitando rotações que prejudiquem o fluxo das veias jugulares.

\section{Discussão}

Para obter resultados positivos em relação aos cuidados críticos de enfermagem associados à DVE e PIC, destaca-se a necessidade de uma avaliação rigorosa da função neurológica através da escala de Glasgow, a mais aplicada na categorização das alterações neurológicas, assim como a identificação de sinais indicativos de infecção, sangramento cerebral e o monitoramento constante do sistema de drenagem (Sakamoto et al., 2020; Cardoso et al., 2018).

Estes cuidados envolvem competências de alta qualidade científica, técnica e humana. Essas habilidades contribuem para estabelecer diagnósticos de enfermagem e intervenções no prognóstico do paciente, no intuito de reduzir o risco de infecção e complicações (Santos et al., 2019).

Para o manejo intensivo de paciente neurocirúrgico pós hemorragia intraparenquimatosa, o enfermeiro deve ter conhecimento para tratar a lesão primária e reconhecer piora neurológica, evitando e tratando o dano neurológico secundário ocasionado pela hipoxemia, aumento do CO2 (hipercapnia), hipo e hipertensão, febre, hipo e hiperglicemia, hiponatremia, convulsão, agitação, tosse e oclusão da DVE de forma inadvertida. Cuidados no posicionamento do paciente são extremamente importantes - a cabeceira do leito deve ser manter um ângulo de $30^{\circ}$, com a finalidade de possibilitar o retorno venoso cerebral pelas veias jugulares, assim como a cabeça deve manter o alinhamento esternal, evitando hiper-extensão ou rotação pois estas reduzem o fluxo na jugular e consequentemente ocasionam um aumento da pressão intracraniana (Souza et al., 2020; AANN, 2018).

Dentre os principais cuidados de enfermagem realizados ao paciente em uso de DVE podemos descrever: suporte exclusivo; checagem do sistema a cada 6 horas; zerar o cateter de DVE no conduto auditivo externo e em caso de obstrução não desobstruir; não aspirar ou ejetar solução pelo cateter; não esquecer de abrir o cateter de DVE depois da realização de qualquer procedimento; cuidados no esvaziamento da sistema coletor da DVE, respeitando capacidade limite; avaliação do aspecto e volume do débito; realização do curativo e avaliação pupilar constante (Witsch et al., 2015; Manzo et al., 2019). 
O enfermeiro deve verificar o correto alinhamento do paciente no leito e, consequentemente, do transdutor de pressão, além das informações do monitor multiparâmetro, como: registro com calibrações periódicas, checagem de todo sistema nos diferentes turnos e manutenção das conexões do sistema de monitorização firmemente unidas. A monitorização da PPC deve ser calculada e mantida em valores igual ou acima de $70 \mathrm{mmHg}$; valores abaixo indicam sinais de hipóxia cerebral e consequentemente morte celular. A mobilização do paciente com DVE no leito é viável e segura quando o paciente estiver estável. (Magalhães et al., 2020; Sakamoto et al., 2021).

Um elevado número de complicações como infecções, desconexão ou obstrução e mau funcionamento do sistema de drenagem podem ser evitados se boas práticas forem adotadas. Neste sentido, profissionais capacitados para realizar intervenções são extremamente fundamentais para o sucesso do tratamento. O uso de bundle para inserção e manutenção do dispositivo é utilizado em alguns centros garantindo equipe qualificada e capacitada para realizar os cuidados (Manzo et al., 2019; Hepburn-Smith et al., 2016).

Uma das limitações deste estudo refere-se a ter sido realizado somente em uma instituição hospitalar da região sul do Brasil, e por tratar-se de um relato de experiência, envolve a percepção dos relatores de forma única e conforme as suas vivências.

\section{Conclusão}

Constata-se a complexidade no cuidado diante do paciente neurocrítico, evidenciando desta forma a necessidade da implantação de intervenções baseadas em planos de cuidados específicos para a obtenção do tratamento adequado. A equipe de enfermagem é a responsável pela manipulação do cateter assim como sua instalação, aferição, adequado posicionamento do paciente no leito e monitorização da PIC, para isso, torna-se evidente a necessidade de competência técnica e científica na busca pela qualificação do cuidado na prática assistencial. Esses achados corroboram para o desfecho de uma assistência de enfermagem baseada em evidências e livre de eventos adversos.

\section{Referências}

Almeida, C. M., Pollo, C. F., \& Meneguin, S. (2019). Nursing interventions for patients with intracranial hypertension: integrative literature review. Aquichan, 19(4), e1949.

American Association of Neuroscience Nurses. (2018). Clinical practice guidelines and evidence-based reviews for neuroscience nurses. Chicago: AANN.

Barcelos, D. G., Santos, C. M., Manhães, L. S. P., \& de Azevedo, A. S. (2016). Atuação do enfermeiro em pacientes vítimas do acidente vascular encefálico hemorrágico na unidade de terapia intensiva. Biológicas \& Saúde, 6(22), 41-53.

Brasil. (2020). Linha de cuidado do acidente vascular cerebral (AVC) no adulto. Brasília: Ministério da Saúde.

Cardoso, A. V. O., Lima, A., Conceição, B. B., Viana, C. L. A., Gonçalves, F. I. R., Torres, J. B., Miranda, L. E. S. M., Morais, L. C. D., Santos, T. L., \& Oliveira, F. B. M. (2017). Uso da Escala de Coma de Glasgow para avaliação do nível de consciência de pacientes com traumatismo crânio encefálico. Revista Eletrônica Acervo Saúde, Sup. 5, S249-S255.

Daltro, M. R., \& Faria, A. A. (2019). Relato de experiência: uma narrativa científica na pós-modernidade. Estudos e Pesquisas em Psicologia, $19(1), 223-237$.

Figueiredo, A. R. G., Pereira, A., \& Mateus, S. (2020). Acidente vascular cerebral isquémico vs hemorrágico: taxa de sobrevivência. Higeia - Revista Científica da Escola Superior de Saúde Dr. Lopes Dias, 3(1), 35-45.

Gonçalves, J., Feitosa, E., \& Borges, R. (2019). Perfil epidemiológico de vítimas de acidente vascular encefálico em um hospital de referência do Ceará/Brasil. Revista Interdisciplinar, 12(2), 92-103.

Guimarães, V. O. S., \& Pereira, C. U. (2018). Acidente vascular cerebral hemorrágico em adultos jovens. JBNC - Jornal Brasileiro de Neurocirurgia, 28(1), 16-20.

Hepburn-Smith, M., Dynkevich, I., Spektor, M., Lord, A., Czeisler, B., \& Lewis, A. (2016). Establishment of an external ventricular drain best practice guideline. Journal of Neuroscience Nursing, 48(1), 54-65.

Magalhães, J. M. P. L., Maciel, C. F., Silva, C. D. B., Melo, J. S., Dias, K. S., Silva, K. O., Damasceno, M.V.S., \& Meneses, S. M. O. C. (2020). Cuidados de enfermagem na manipulação do cateter de DVE e PIC através do relato de um caso clínico. Brazilian Journal of Health Review, 3(5), $15243-15252$. 
Research, Society and Development, v. 10, n. 10, e355101018715, 2021

(CC BY 4.0) | ISSN 2525-3409 | DOI: http://dx.doi.org/10.33448/rsd-v10i10.18715

Manzo, B. F., Macedo, K. O., Barbosa, L. M., Simão, D., Souza, R. S., Correa, A. R., \& Costa, A. C. L. (2019). Repercussão de intervenção educativa no conhecimento da equipe de enfermagem sobre os cuidados no uso da derivação ventricular externa em pediatria. REME - Revista Mineira de Enfermagem, 23, e1189.

Minayo, M. C. S. (2012). Análise qualitativa: teoria, passos e fidedignidade. Ciência \& Saúde Coletiva, 17(3), 621-626.

Nascimento, D. S., \& Carvalho, M. M. B. (2020). Perfil epidemiológico de pacientes com acidente vascular cerebral no sertão central. Encontro de Extensão, Docência e Iniciação Científica (EEDIC), 7.

Rogers, M., Stutzman, S. E., Atem, F. D., Sengupta, S., Welch, B., \& Olson, D. M. (2017). Intracranial pressure values are highly variable after cerebral spinal fluid drainage. Journal of Neuroscience Nursing, 49(2), 85-89.

Sakamoto, V. T. M., Vieira, T. W., Viegas, K., Blatt, C. R., \& Caregnato, R. C. A. (2021). Cuidados de enfermagem na assistência ao paciente com derivação ventricular externa: scoping review. Revista Brasileira de Enfermagem, 74(2), e20190796.

Santos, L. N. C., Aquino, R. G., Souza, A. P., Silva, N. C. M., \& Luna, A. A. (2019). Diagnósticos de enfermagem em pós-operatório de neurocirurgia. Revista de Enfermagem da UFPE on line, 13, 1-12.

Souza, R. C. S., Siqueira, E. M. P., Meira, L., Araujo, G. L., \& Bersaneti, M. D. (2020). Retenção de conhecimento dos enfermeiros sobre derivação ventricular externa. Revista Cuidarte, 11(1), e784.

Szabo, C. M., Grap, M. J., Munro, C. L., Starkweather, A. M., \& Randall, E. (2014). The effect of oral care on intracranial pressure in critically ill adults. The Journal of Neuroscience Nursing, 46(6), 321-329.

Witsch, J., Bruce, E., Meyers, E., Velazquez, A., Schmidt, M., Suwatcharangkoon, S., Sachin, A., Soojin, P., Falo, C. E., Connolly, S., \& Claassen, J. (2015). Intraventricular hemorrhage expansion in patients with spontaneous intracerebral hemorrhage. Neurology, 84(10), 989-994. 